\title{
Hermite Interpolation on the Unit Circle Considering up to the Second Derivative
}

\author{
Elías Berriochoa, ${ }^{1}$ Alicia Cachafeiro, ${ }^{2}$ and Jaime Díaz ${ }^{2}$ \\ ${ }^{1}$ Departamento de Matemática Aplicada I, Facultad de Ciencias, Universidad de Vigo, 32004 Ourense, Spain \\ ${ }^{2}$ Departamento de Matemática Aplicada I, E. Ingeniería Industrial, Universidad de Vigo, 36310 Vigo, Spain
}

Correspondence should be addressed to Alicia Cachafeiro; acachafe@uvigo.es

Received 18 December 2013; Accepted 8 January 2014; Published 10 March 2014

Academic Editors: G. Ólafsson and T. Tran

Copyright (C) 2014 Elías Berriochoa et al. This is an open access article distributed under the Creative Commons Attribution License, which permits unrestricted use, distribution, and reproduction in any medium, provided the original work is properly cited.

\begin{abstract}
The paper is devoted to study the Hermite interpolation problem on the unit circle. The interpolation conditions prefix the values of the polynomial and its first two derivatives at the nodal points and the nodal system is constituted by complex numbers equally spaced on the unit circle. We solve the problem in the space of Laurent polynomials by giving two different expressions for the interpolation polynomial. The first one is given in terms of the natural basis of Laurent polynomials and the remarkable fact is that the coefficients can be computed in an easy and efficient way by means of the Fast Fourier Transform (FFT). The second expression is a barycentric formula, which is very suitable for computational purposes.
\end{abstract}

\section{Introduction}

One of the pioneering papers concerning Hermite interpolation on the unit circle is [1]. There a Fejér's type theorem is proved (see $[2,3]$ ), for nodal systems constituted by the $n$ roots of a complex number with modulus one. The main result asserts that the Hermite-Fejér interpolants uniformly converge for continuous functions on the unit circle. Some improvements to this result, considering nonvanishing derivatives and more smooth functions, were given in [4]. More recently, in [5], the order of convergence of HermiteFejér interpolants for analytic functions on a disk and on an annulus containing the unit circle was obtained.

The classical Hermite interpolation on the circle with nodal points equally spaced was studied in [6]. There it was constructed an orthogonal basis for the space of polynomials in order to obtain the expression of the interpolation polynomials. The coefficients of the interpolation polynomials in this basis can be computed by using the FFT. In [7], the same problem was studied and the corresponding expressions for the Laurent polynomials of interpolation were obtained in a more simple way. Another basis was constructed and again the coefficients can be computed by using the FFT. From these formulas, suitable expressions for the fundamental polynomials were obtained and the barycentric formulas for Hermite interpolation on the unit circle were deduced for the first time. The barycentric formulas were known for Hermite interpolation on the bounded interval (see [8]), but [7] was a new contribution on the circle.

A study about Hermite interpolation on two disjoint sets of nodes on the unit circle has been developed in [9] and problems considering more than one derivative were also considered. Indeed, lacunary Hermite interpolation problems have been also studied on some nonuniformly distributed nodes on the unit circle (see [10]).

In the present paper we study generalized Hermite interpolation problems on the unit circle considering nodal points equally spaced and using the values for the first two derivatives. First we obtain suitable basis for subspaces of the space of Laurent polynomials by considering appropriate interpolation conditions. This enables us to express the interpolation polynomials in such a way that the coefficients can be computed by using the FFT.

In the second part of the paper we deduce the barycentric formulas which constitute a new contribution of the paper. Like in the Lagrange interpolation (see [11]), the barycentric expressions are very useful for doing evaluations and calculus due to their stability (see [12]). 


\section{Laurent Hermite Interpolation Polynomials}

We study the generalized Hermite interpolation problem on the unit circle $\mathbb{T}:=\{z:|z|=1\}$ considering the first two derivatives. The nodal system $\left\{\alpha_{j}\right\}_{j=0}^{n-1}$ is constituted by the $n$ roots of a complex number $\lambda$, with $|\lambda|=1$; that is, it consists of complex numbers equally spaced on the unit circle. The problem to solve can be posed as follows.

If $p(n)$ and $q(n)$ are two nondecreasing sequences of nonnegative integers such that $p(n)+q(n)=3 n-1, n=$ $1,2, \ldots$, find the unique Laurent polynomial $\mathscr{H}_{-p(n), q(n)}(z) \in$ $\Lambda_{-p(n), q(n)}[z]=\operatorname{span}\left\{z^{k}:-p(n) \leq k \leq q(n)\right\}$ satisfying the interpolation conditions

$$
\begin{array}{r}
\mathscr{H}_{-p(n), q(n)}\left(\alpha_{j}\right)=u_{j}, \\
\mathscr{H}_{-p(n), q(n)}^{\prime}\left(\alpha_{j}\right)=v_{j}, \\
\mathscr{H}_{-p(n), q(n)}^{\prime \prime}\left(\alpha_{j}\right)=w_{j} \\
j=0, \cdots, n-1,
\end{array}
$$

where $\left\{u_{j}\right\}_{j=0}^{n-1},\left\{v_{j}\right\}_{j=0}^{n-1}$ and $\left\{w_{j}\right\}_{j=0}^{n-1}$ are prefixed complex numbers.

For simplicity and without loss of generality we consider the case when $p(n)=n+E[n / 2]$ and $q(n)=n+E[(n-$ $1) / 2]$ and we denote the polynomial $\mathscr{H}_{-n-E[n / 2], n+E[(n-1) / 2]}(z)$ by $\mathscr{H}(z)$.

To obtain an expression of the interpolation polynomial, first we introduce some auxiliary polynomials belonging to the Laurent space $\Lambda_{-n-E[n / 2], n+E[(n-1) / 2]}[z]$ that we denote by $\mathscr{L}_{0, k}, \mathscr{L}_{1, k}$, and $\mathscr{L}_{2, k}$, with $-E(n / 2) \leqslant k \leqslant E[(n-1) / 2]$, and which are characterized by the following interpolation conditions:

$$
\begin{gathered}
\mathscr{L}_{0, k}\left(\alpha_{j}\right)=\alpha_{j}^{k}, \quad \mathscr{L}_{0, k}^{\prime}\left(\alpha_{j}\right)=0, \\
\mathscr{L}_{0, k}^{\prime \prime}\left(\alpha_{j}\right)=0 \quad \text { for } j=0, \ldots, n-1, \\
\mathscr{L}_{1, k}\left(\alpha_{j}\right)=0, \quad \mathscr{L}_{1, k}^{\prime}\left(\alpha_{j}\right)= \begin{cases}\alpha_{j}^{-1} & \text { if } k=0, \\
k \alpha_{j}^{k-1} & \text { if } k \neq 0,\end{cases} \\
\mathscr{L}_{1, k}^{\prime \prime}\left(\alpha_{j}\right)=0 \quad \text { for } j=0, \ldots, n-1, \\
\mathscr{L}_{2, k}\left(\alpha_{j}\right)=0, \quad \mathscr{L}_{2, k}^{\prime}\left(\alpha_{j}\right)=0, \\
\mathscr{L}_{2, k}^{\prime \prime}\left(\alpha_{j}\right)= \begin{cases}\alpha_{j}^{-2} & \text { if } k=0, \\
\alpha_{j}^{-1} & \text { if } k=1, \\
k(k-1) \alpha_{j}^{k-2} & \text { if } k \notin\{0,1\},\end{cases}
\end{gathered}
$$

for $j=0, \ldots, n-1$.
Proposition 1. For $-E(n / 2) \leqslant k \leqslant E[(n-1) / 2]$, the polynomials $\mathscr{L}_{0, k}(z), \mathscr{L}_{1, k}(z)$, and $\mathscr{L}_{2, k}(z)$ satisfying (2), (3), and (4), respectively, have the following expressions:

$$
\begin{aligned}
& \mathscr{L}_{0, k}(z) \\
& =\frac{1}{n^{2}}\left[\frac{\lambda}{2} k(k+n) z^{k-n}+\left(n^{2}-k^{2}\right) z^{k}+\frac{k}{2 \lambda}(k-n) z^{n+k}\right], \\
& \mathscr{L}_{1, k}(z) \\
& = \begin{cases}\frac{1}{n^{2}}\left[\frac{\lambda(1-n)}{2} z^{-n}-1+\frac{n+1}{2 \lambda} z^{n}\right] & \text { if } k=0, \\
\frac{1}{n^{2}}\left[-\frac{\lambda}{2} k(n+2 k-1) z^{k-n}+k(2 k-1) z^{k}\right. & \text { if } k \neq 0, \\
& \left.+\frac{k}{2 \lambda}(n-2 k+1) z^{n+k}\right]\end{cases} \\
& \mathscr{L}_{2, k}(z) \quad \text { if } k=0, \\
& = \begin{cases}\frac{1}{n^{2}}\left[\frac{\lambda}{2} z^{-n}-1+\frac{1}{2 \lambda} z^{n}\right] \\
\frac{1}{n^{2}}\left[\frac{\lambda}{2} z^{1-n}-z+\frac{1}{2 \lambda} z^{n+1}\right] & \text { if } k=1, \\
\frac{1}{n^{2}}\left[\frac{\lambda}{2} k(k-1) z^{k-n}+k(1-k) z^{k}\right. & \text { if } k \notin 0,1\} . \\
\left.+\frac{1}{2 \lambda} k(k-1) z^{n+k}\right] & \end{cases}
\end{aligned}
$$

Proof. The polynomial that interpolates $z^{k}$ satisfying (2) has the following form:

$$
\mathscr{L}_{0, k}(z)={ }_{0} a_{k} z^{k}+{ }_{0} b_{k} z^{n+k}+{ }_{0} c_{k} z^{k-n} .
$$

By applying the first interpolation condition we get that ${ }_{0} a_{k}+$ ${ }_{0} b_{k} \lambda+{ }_{0} c_{k}(1 / \lambda)=1$ and by using the second interpolation condition we have ${ }_{0} a_{k} k+{ }_{0} b_{k}(n+k) \lambda+{ }_{0} c_{k}(k-n)(1 / \lambda)=0$. Taking again derivatives and applying the third interpolation condition we obtain ${ }_{0} a_{k} k(k-1)+{ }_{0} b_{k}(n+k)(n+k-1) \lambda+$ ${ }_{0} c_{k}(k-n)(k-n-1)(1 / \lambda)=0$. Thus we have a linear system in the unknowns ${ }_{0} a_{k},{ }_{0} b_{k}$, and ${ }_{0} c_{k}$, which has the following solutions ${ }_{0} a_{k}=\left(1 / n^{2}\right)\left(n^{2}-k^{2}\right),{ }_{0} b_{k}=\left(k / 2 n^{2} \lambda\right)(k-n)$, and ${ }_{0} c_{k}=\left(k \lambda / 2 n^{2}\right)(k+n)$, from which we obtain the expression of $\mathscr{L}_{0, k}(z)$.

The polynomial $\mathscr{L}_{1, k}(z)$ satisfying (3) has the form

$$
\mathscr{L}_{1, k}(z)={ }_{1} a_{k} z^{k}+{ }_{1} b_{k} z^{n+k}+{ }_{1} c_{k} z^{k-n} .
$$

By imposing the first interpolation condition we get ${ }_{1} a_{k}+{ }_{1} b_{k} \lambda+{ }_{1} c_{k}(1 / \lambda)=0$.

If we assume that $k \neq 0$, we take derivatives and use the second interpolation condition then we obtain ${ }_{1} a_{k} k+{ }_{1} b_{k}(n+$ $k) \lambda+{ }_{1} c_{k}(k-n)(1 / \lambda)=k$. By taking again derivatives and using the third interpolation condition we have ${ }_{1} a_{k} k(k-1)+$ ${ }_{1} b_{k}(n+k)(n+k-1) \lambda+{ }_{1} c_{k}(k-n)(k-n-1)(1 / \lambda)=0$. Hence we have a system with unknowns ${ }_{1} a_{k},{ }_{1} b_{k}$, and ${ }_{1} c_{k}$, which has the following solutions: ${ }_{1} a_{k}=\left(k / n^{2}\right)(2 k-1),{ }_{1} b_{k}=$ $\left(k / 2 n^{2} \lambda\right)(n-2 k+1)$, and ${ }_{1} c_{k}=-\left(k \lambda / 2 n^{2}\right)(n+2 k-1)$. 
If $k=0$ then $\mathscr{L}_{1,0}^{\prime}(z)={ }_{1} b_{0} n z^{n-1}-{ }_{1} c_{0} n z^{-n-1}$. Thus if we evaluate at $\alpha_{j}$ and apply the second interpolation condition we get ${ }_{1} b_{0} n \lambda-{ }_{1} c_{0} n(1 / \lambda)=1$. By taking again derivatives and using the third interpolation condition we have ${ }_{1} b_{0} n(n-$ 1) $\lambda+{ }_{1} c_{0} n(n+1)(1 / \lambda)=0$. Then, if we solve the corresponding system we have that ${ }_{1} a_{0}=-1 / n^{2},{ }_{1} b_{0}=\left(1 / 2 n^{2} \lambda\right)(n+1)$ and ${ }_{1} c_{0}=\left(\lambda / 2 n^{2}\right)(1-n)$. Hence we obtain the expression for $\mathscr{L}_{1, k}(z)$ given in our statement.

Finally, the polynomial $\mathscr{L}_{2, k}(z)$ satisfying (4) can be written as

$$
\mathscr{L}_{2, k}(z)={ }_{2} a_{k} z^{k}+{ }_{2} b_{k} z^{n+k}+{ }_{2} c_{k} z^{k-n} .
$$

By applying the first interpolation condition we obtain ${ }_{2} a_{k}+{ }_{2} b_{k} \lambda+{ }_{2} c_{k}(1 / \lambda)=0$ and if we use the second interpolation condition we get ${ }_{2} a_{k} k+{ }_{2} b_{k}(n+k) \lambda+{ }_{2} c_{k}(k-$ $n)(1 / \lambda)=0$.

Now if we assume that $k \notin\{0,1\}$ and use the third interpolation condition we obtain ${ }_{2} a_{k} k(k-1)+{ }_{2} b_{k}(n+k)(n+$ $k-1) \lambda+{ }_{2} c_{k}(k-n)(k-n-1)(1 / \lambda)=k(k-1)$. Then, if we solve the corresponding system we have ${ }_{2} a_{k}=\left(k / n^{2}\right)(1-k)$, ${ }_{2} b_{k}=\left(k / 2 n^{2} \lambda\right)(k-1)$, and ${ }_{2} c_{k}=\left(\lambda k / 2 n^{2}\right)(k-1)$.

If $k=0$ and we use the third interpolation condition we obtain ${ }_{2} b_{0} n(n-1) \lambda+{ }_{2} c_{0} n(n+1)(1 / \lambda)=1$. By solving the system we have the following solution: ${ }_{2} a_{0}=-1 / n^{2},{ }_{2} b_{0}=$ $1 / 2 n^{2} \lambda$, and ${ }_{2} \mathcal{C}_{0}=\lambda / 2 n^{2}$.

Finally, if $k=1$ then ${ }_{2} b_{1} n(n+1) \lambda+{ }_{2} c_{1} n(n-1)(1 / \lambda)=$ 1 . By solving the corresponding system in the unknowns ${ }_{2} a_{1},{ }_{2} b_{1}$, and ${ }_{2} c_{1}$ we obtain that ${ }_{2} a_{1}=-1 / n^{2},{ }_{2} b_{1}=1 / 2 n^{2} \lambda$, and ${ }_{2} c_{1}=\lambda / 2 n^{2}$.

Next we prove that these auxiliary polynomials constitute a suitable basis of the space $\Lambda_{-n-E[n / 2], n+E[(n-1) / 2]}[z]$.

Proposition 2. The system

$$
\left\{\mathscr{L}_{0, k}(z)\right\}_{k=-E[n / 2]}^{E[(n-1) / 2]} \cup\left\{\mathscr{L}_{1, k}(z)\right\}_{k=-E[n / 2]}^{E[(n-1) / 2]} \cup\left\{\mathscr{L}_{2, k}(z)\right\}_{k=-E[n / 2]}^{E[(n-1) / 2]}
$$

is an orthogonal basis of the Laurent space $\Lambda_{-n-E[n / 2], n+E[(n-1) / 2]}[z]$ with respect to the inner product defined by

$\langle P, Q\rangle$

$$
=\frac{1}{n} \sum_{j=0}^{n-1}\left(P\left(\alpha_{j}\right) \overline{Q\left(\alpha_{j}\right)}+P^{\prime}\left(\alpha_{j}\right) \overline{Q^{\prime}\left(\alpha_{j}\right)}+P^{\prime \prime}\left(\alpha_{j}\right) \overline{Q^{\prime \prime}\left(\alpha_{j}\right)}\right)
$$

for $P, Q \in \Lambda_{-n-E[n / 2], n+E[(n-1) / 2]}[z]$.

Moreover, it holds

$$
\begin{gathered}
\left\|\mathscr{L}_{0, k}(z)\right\|^{2}=1, \quad\left\|\mathscr{L}_{1, k}(z)\right\|^{2}= \begin{cases}1, & \text { if } k=0, \\
k^{2}, & \text { if } k \neq 0,\end{cases} \\
\left\|\mathscr{L}_{2, k}(z)\right\|^{2}= \begin{cases}1, & \text { if } k \in\{0,1\}, \\
k^{2}(k-1)^{2}, & \text { if } k \notin\{0,1\} .\end{cases}
\end{gathered}
$$

Proof. It is easy to check that

$$
\begin{aligned}
& \left\langle\mathscr{L}_{0, k}(z), \mathscr{L}_{0, l}(z)\right\rangle \\
& =\frac{1}{n} \sum_{j=0}^{n-1}\left(\mathscr{L}_{0, k}\left(\alpha_{j}\right) \overline{\mathscr{L}_{0, l}\left(\alpha_{j}\right)}+\mathscr{L}_{0, k}^{\prime}\left(\alpha_{j}\right) \overline{\mathscr{L}_{0, l}^{\prime}\left(\alpha_{j}\right)}\right. \\
& \left.+\mathscr{L}_{0, k}^{\prime \prime}\left(\alpha_{j}\right) \overline{\mathscr{L}_{0, l}^{\prime \prime}\left(\alpha_{j}\right)}\right) \\
& =\frac{1}{n} \sum_{j=0}^{n-1} \alpha_{j}^{k} \alpha_{j}^{\bar{l}}= \begin{cases}1, & \text { if } k=l, \\
0, & \text { otherwise, }\end{cases} \\
& \left\langle\mathscr{L}_{0, k}(z), \mathscr{L}_{1, l}(z)\right\rangle \\
& =\frac{1}{n} \sum_{j=0}^{n-1}\left(\mathscr{L}_{0, k}\left(\alpha_{j}\right) \overline{\mathscr{L}_{1, l}\left(\alpha_{j}\right)}+\mathscr{L}_{0, k}^{\prime}\left(\alpha_{j}\right) \overline{\mathscr{L}_{1, l}^{\prime}\left(\alpha_{j}\right)}\right. \\
& \left.+\mathscr{L}_{0, k}^{\prime \prime}\left(\alpha_{j}\right) \overline{\mathscr{L}_{1, l}^{\prime \prime}\left(\alpha_{j}\right)}\right)=0, \\
& \left\langle\mathscr{L}_{0, k}(z), \mathscr{L}_{2, l}(z)\right\rangle \\
& =\frac{1}{n} \sum_{j=0}^{n-1}\left(\mathscr{L}_{0, k}\left(\alpha_{j}\right) \overline{\mathscr{L}_{2, l}\left(\alpha_{j}\right)}+\mathscr{L}_{0, k}^{\prime}\left(\alpha_{j}\right) \overline{\mathscr{L}_{2, l}^{\prime}\left(\alpha_{j}\right)}\right. \\
& \left.+\mathscr{L}_{0, k}^{\prime \prime}\left(\alpha_{j}\right) \overline{\mathscr{L}_{2, l}^{\prime \prime}\left(\alpha_{j}\right)}\right)=0, \\
& \left\langle\mathscr{L}_{1, k}(z), \mathscr{L}_{1, l}(z)\right\rangle \\
& =\frac{1}{n} \sum_{j=0}^{n-1}\left(\mathscr{L}_{1, k}\left(\alpha_{j}\right) \overline{\mathscr{L}_{1, l}\left(\alpha_{j}\right)}+\mathscr{L}_{1, k}\left(\alpha_{j}\right) \overline{\mathscr{L}_{1, l}^{\prime}\left(\alpha_{j}\right)}\right. \\
& \left.+\mathscr{L}_{1, k}^{\prime \prime}\left(\alpha_{j}\right) \overline{\mathscr{L}_{1, l}^{\prime \prime}\left(\alpha_{j}\right)}\right) \\
& = \begin{cases}1, & \text { if } k=l=0, \\
k^{2}, & \text { if } k=l \neq 0, \\
0, & \text { otherwise, }\end{cases} \\
& \left\langle\mathscr{L}_{1, k}(z), \mathscr{L}_{2, l}(z)\right\rangle \\
& =\frac{1}{n} \sum_{j=0}^{n-1}\left(\mathscr{L}_{1, k}\left(\alpha_{j}\right) \overline{\mathscr{L}_{2, l}\left(\alpha_{j}\right)}+\mathscr{L}_{1, k}^{\prime}\left(\alpha_{j}\right) \overline{\mathscr{L}_{2, l}^{\prime}\left(\alpha_{j}\right)}\right. \\
& \left.+\mathscr{L}_{1, k}^{\prime \prime}\left(\alpha_{j}\right) \overline{\mathscr{L}_{2, l}^{\prime \prime}\left(\alpha_{j}\right)}\right)=0, \\
& \left\langle\mathscr{L}_{2, k}(z), \mathscr{L}_{2, l}(z)\right\rangle \\
& =\frac{1}{n} \sum_{j=0}^{n-1}\left(\mathscr{L}_{2, k}\left(\alpha_{j}\right) \overline{\mathscr{L}_{2, l}\left(\alpha_{j}\right)}+\mathscr{L}_{2, k}^{\prime}\left(\alpha_{j}\right) \overline{\mathscr{L}_{2, l}^{\prime}\left(\alpha_{j}\right)}\right. \\
& \left.+\mathscr{L}_{1, k}^{\prime \prime}\left(\alpha_{j}\right) \overline{\mathscr{L}_{2, l}^{\prime \prime}\left(\alpha_{j}\right)}\right) \\
& =\frac{1}{n} \sum_{j=0}^{n-1} k(k-1) \alpha_{j}^{k-2} l(l-1){\overline{\alpha_{j}}}^{l-2}
\end{aligned}
$$




$$
\begin{aligned}
& =\frac{1}{n} \sum_{j=0}^{n-1} k(k-1) l(l-1) \alpha_{j}^{k-l} \\
& = \begin{cases}1, & \text { if } k=l \text {, with } l \in\{0,1\}, \\
k^{2}(k-1)^{2}, & \text { if } k=l, \text { with } l \notin\{0,1\}, \\
0, & \text { otherwise. }\end{cases}
\end{aligned}
$$

Now we are in conditions to obtain the expression of the polynomial $\mathscr{H}_{-n-E[n / 2], n+E[(n-1) / 2]}(z)$ satisfying $(1)$ that we denote for simplicity $\mathscr{H}(z)$. It is clear that it can be written as

$$
\mathscr{H}=\mathscr{H}_{0}+\mathscr{H}_{1}+\mathscr{H}_{2},
$$

where $\mathscr{H}_{0}, \mathscr{H}_{1}$, and $\mathscr{H}_{2}$ are the solutions of the following problems:

$$
\begin{aligned}
& \mathscr{H}_{0} \in \Lambda_{-n-E[n / 2], n+E[(n-1) / 2]}[z] \text { and it satisfies } \\
& \mathscr{H}_{0}\left(\alpha_{j}\right)=u_{j}, \mathscr{H}_{0}^{\prime}\left(\alpha_{j}\right)=0, \text { and } \mathscr{H}_{0}^{\prime \prime}\left(\alpha_{j}\right)=0 \text { for } \\
& j=0, \ldots, n-1 . \\
& \mathscr{H}_{1} \in \Lambda_{-n-E[n / 2], n+E[(n-1) / 2]}[z] \text { and it satisfies } \\
& \mathscr{H}_{1}\left(\alpha_{j}\right)=0, \mathscr{H}_{1}^{\prime}\left(\alpha_{j}\right)=v_{j} \text {, and } \mathscr{H}_{1}^{\prime \prime}\left(\alpha_{j}\right)=0 \text { for } j= \\
& 0, \ldots, n-1 . \\
& \mathscr{H}_{2} \in \Lambda_{-n-E[n / 2], n+E[(n-1) / 2]}[z] \text { and it satisfies } \\
& \mathscr{H}_{2}\left(\alpha_{j}\right)=0, \mathscr{H}_{2}^{\prime}\left(\alpha_{j}\right)=0 \text {, and } \mathscr{H}_{2}^{\prime \prime}\left(\alpha_{j}\right)=w_{j} \text { for } j= \\
& 0, \ldots, n-1 .
\end{aligned}
$$

Proposition 3. The polynomials $\mathscr{H}_{0}, \mathscr{H}_{1}, \mathscr{H}_{2}$, and $\mathscr{H}$ defined before have the following expressions:

(i)

$$
\begin{aligned}
\mathscr{H}_{0}(z)=\frac{1}{n^{2}} & \sum_{k=-E[n / 2]}^{E[(n-1) / 2]}\left(\frac{1}{n} \sum_{j=0}^{n-1} u_{j} \bar{\alpha}_{j}^{k}\right) \\
\times & {\left[\frac{\lambda}{2} k(k+n) z^{k-n}+\left(n^{2}-k^{2}\right) z^{k}\right.} \\
& \left.+\frac{k}{2 \lambda}(k-n) z^{n+k}\right],
\end{aligned}
$$

(ii)

$$
\begin{aligned}
\mathscr{H}_{1}(z)= & \frac{1}{n^{2}} \sum_{k=-E[n / 2]}^{E[(n-1) / 2]}\left(\frac{1}{n} \sum_{j=0}^{n-1} v_{j} \bar{\alpha}_{j}^{k-1}\right) \\
\times & {\left[-\frac{\lambda}{2}(n+2 k-1) z^{k-n}+(2 k-1) z^{k}\right.} \\
& \left.+\frac{1}{2 \lambda}(n-2 k+1) z^{n+k}\right],
\end{aligned}
$$

(iii)

$$
\begin{aligned}
\mathscr{H}_{2}(z)= & \frac{1}{n^{2}} \sum_{k=-E[n / 2]}^{E[(n-1) / 2]}\left(\frac{1}{n} \sum_{j=0}^{n-1} w_{j}{\overline{\alpha_{j}}}^{k-2}\right) \\
& \times\left[\frac{\lambda}{2} z^{k-n}-z^{k}+\frac{1}{2 \lambda} z^{n+k}\right],
\end{aligned}
$$

(iv)

$H(z)$

$$
\begin{aligned}
& =\frac{1}{n^{3}} \sum_{k=-E[n / 2]}^{E[(n-1) / 2]}\left\{\frac { \lambda } { 2 } \left[\left(\sum_{j=0}^{n-1} u_{j} \bar{\alpha}_{j}^{k}\right) k(k+n)\right.\right. \\
& -\left(\sum_{j=0}^{n-1} v_{j}{\overline{\alpha_{j}}}^{k-1}\right)(n+2 k-1) \\
& \left.+\left(\sum_{j=0}^{n-1} w_{j}{\overline{\alpha_{j}}}^{k-2}\right)\right] z^{k-n} \\
& +\left[\left(\sum_{j=0}^{n-1} u_{j}{\overline{\alpha_{j}}}^{k}\right)\left(n^{2}-k^{2}\right)+\left(\sum_{j=0}^{n-1} v_{j}{\overline{\alpha_{j}}}^{k-1}\right)\right. \\
& \left.\times(2 k-1)-\left(\sum_{j=0}^{n-1} w_{j} \bar{\alpha}_{j}^{k-2}\right)\right] z^{k} \\
& +\frac{1}{2 \lambda}\left[\left(\sum_{j=0}^{n-1} u_{j}{\overline{\alpha_{j}}}^{k}\right) k(k-n)\right. \\
& +\left(\sum_{j=0}^{n-1} v_{j}{\overline{\alpha_{j}}}^{k-1}\right)(n-2 k+1) \\
& \left.\left.+\left(\sum_{j=0}^{n-1} w_{j} \bar{\alpha}_{j}^{k-2}\right)\right] z^{n+k}\right\} .
\end{aligned}
$$

Proof. (i) It is clear that $\mathscr{H}_{0}(z)$ can be written as

$$
\mathscr{H}_{0}(z)=\sum_{k=-E[n / 2]}^{E[(n-1) / 2]} a_{k} \mathscr{L}_{0, k}(z)
$$

For computing the coefficients we take into account that $\left\langle\mathscr{H}_{0}(z), \mathscr{L}_{0, k}(z)\right\rangle=a_{k}\left\|\mathscr{L}_{0, k}(z)\right\|^{2}=a_{k}$. On the other hand, it holds that $\left\langle\mathscr{H}_{0}(z), \mathscr{L}_{0, k}(z)\right\rangle=(1 / n) \sum_{j=0}^{n-1} u_{j} \bar{\alpha}_{j}^{k}$ and therefore $a_{k}=(1 / n) \sum_{j=0}^{n-1} u_{j} \bar{\alpha}_{j}{ }^{k}$. Hence, using the expression of $\mathscr{L}_{0, k}(z)$, we have (i).

(ii) Proceeding in the same way, $\mathscr{H}_{1}(z)$ can be written as

$$
\mathscr{H}_{1}(z)=\sum_{k=-E[n / 2]}^{E[(n-1) / 2]} b_{k} \mathscr{L}_{1, k}(z)
$$

On one hand, if we calculate the inner product we have

$$
\left\langle\mathscr{H}_{1}(z), \mathscr{L}_{1, k}(z)\right\rangle=b_{k}\left\|\mathscr{L}_{1, k}(z)\right\|^{2}= \begin{cases}b_{0}, & \text { if } k=0 \\ b_{k} k^{2}, & \text { otherwise }\end{cases}
$$


On the other hand, it holds that

$$
\frac{1}{n} \sum_{j=0}^{n-1} \mathscr{H}_{1}^{\prime}\left(\alpha_{j}\right) \overline{\mathscr{L}_{1, k}^{\prime}\left(\alpha_{j}\right)}= \begin{cases}\frac{1}{n} \sum_{j=0}^{n-1} v_{j}{\overline{\alpha_{j}}}^{-1}, & \text { if } k=0, \\ \frac{1}{n} \sum_{j=0}^{n-1} k v_{j}{\overline{\alpha_{j}}}^{k-1}, & \text { otherwise }\end{cases}
$$

and therefore

$$
b_{k}= \begin{cases}\frac{1}{n} \sum_{j=0}^{n-1} v_{j}{\overline{\alpha_{j}}}^{-1}, & \text { if } k=0 \\ \frac{1}{n k} \sum_{j=0}^{n-1} v_{j} \bar{\alpha}_{j}^{k-1}, & \text { otherwise. }\end{cases}
$$

Hence, taking into account the expression of $\mathscr{L}_{1, k}(z)$, we obtain (ii).

(iii) We write $\mathscr{H}_{2}(z)$ as follows

$$
\mathscr{H}_{2}(z)=\sum_{k=-E[n / 2]}^{E[(n-1) / 2]} c_{k} \mathscr{L}_{2, k}(z) .
$$

By computing the inner product, we obtain

$$
\begin{aligned}
& \left\langle\mathscr{H}_{2}(z), \mathscr{L}_{2, k}(z)\right\rangle=c_{k}\left\|\mathscr{L}_{2, k}(z)\right\|^{2} \\
& = \begin{cases}c_{k}, & \text { if } k \in\{0,1\}, \\
c_{k} k^{2}(k-1)^{2}, & \text { otherwise, }\end{cases} \\
& \frac{1}{n} \sum_{j=0}^{n-1} \mathscr{H}_{2}^{\prime \prime}\left(\alpha_{j}\right) \overline{\mathscr{L}_{2, k}^{\prime \prime}\left(\alpha_{j}\right)} \\
& = \begin{cases}\frac{1}{n} \sum_{j=0}^{n-1} w_{j} \bar{\alpha}_{j}^{-2}, & \text { if } k=0, \\
\frac{1}{n} \sum_{j=0}^{n-1} w_{j} \bar{\alpha}_{j}^{-1}, & \text { if } k=1, \\
\frac{1}{n} \sum_{j=0}^{n-1} w_{j} k(k-1) \bar{\alpha}_{j}^{k-2}, & \text { otherwise. }\end{cases}
\end{aligned}
$$

Therefore

$$
c_{k}= \begin{cases}\frac{1}{n} \sum_{j=0}^{n-1} w_{j}{\overline{\alpha_{j}}}^{-2}, & \text { if } k=0, \\ \frac{1}{n} \sum_{j=0}^{n-1} w_{j}{\overline{\alpha_{j}}}^{-1}, & \text { if } k=1, \\ \frac{1}{k(k-1) n} \sum_{j=0}^{n-1} w_{j}{\overline{\alpha_{j}}}^{k-2}, & \text { otherwise }\end{cases}
$$

and we obtain the expression of $\mathscr{L}_{2, k}(z)$ given in (iii).

(iv) It is straightforward from

$$
\mathscr{H}(z)=\mathscr{H}_{0}(z)+\mathscr{H}_{1}(z)+\mathscr{H}_{2}(z) .
$$

Next we consider the particular cases in which the nodes are $n$ roots of 1 and -1 , obtaining the following results.

Corollary 4. (i) If $\left\{y_{j}\right\}_{j=0}^{2 n-1}$ are the $2 n$ roots of -1 , then the Hermite interpolation polynomial satisfying (1) is

$$
\begin{aligned}
\mathscr{H}_{-3 n, 3 n-1}(z) \frac{1}{8 n^{3}} \sum_{k=-n}^{n-1}\left\{\frac{-1}{2}[\right. & \left(\sum_{j=0}^{2 n-1} u_{j} \bar{y}_{j}^{k}\right) k(k+2 n) \\
& -\left(\sum_{j=0}^{2 n-1} v_{j} \bar{y}_{j}^{k-1}\right)(2 n+2 k-1) \\
& \left.+\left(\sum_{j=0}^{2 n-1} w_{j} \bar{y}_{j}^{k-2}\right)\right] z^{k-2 n} \\
+ & \left(\sum_{j=0}^{2 n-1} u_{j} \bar{y}_{j}^{k}\right)\left(4 n^{2}-k^{2}\right) \\
& +\left(\sum_{j=0}^{2 n-1} v_{j} \bar{y}_{j}^{k-1}\right)(2 k-1) \\
- & \left.\left(\sum_{j=0}^{2 n-1} w_{j} \bar{y}_{j}^{k-2}\right)\right] z^{k} \\
& {\left[\left(\sum_{j=0}^{2 n-1} u_{j} \bar{y}_{j}^{k}\right)_{j=0}^{2 n-1} v_{j} \bar{y}_{j}^{k-1}\right)(2 n-2 k+1) }
\end{aligned}
$$

(ii) If $\left\{y_{j}\right\}_{j=0}^{2 n}$ are the $2 n+1$ roots of -1 , then the Hermite interpolation polynomial satisfying (1) is

$$
\begin{aligned}
\mathscr{H}_{-3 n-1,3 n+1}(z) & \frac{1}{(2 n+1)^{3}} \\
= & \quad \sum_{k=-n}^{n}\left\{\frac { - 1 } { 2 } \left[\left(\sum_{j=0}^{2 n} u_{j} \bar{y}_{j}^{k}\right) k(k+2 n+1)\right.\right. \\
& \quad-\left(\sum_{j=0}^{2 n} v_{j} \bar{y}_{j}^{k-1}\right)(2 n+2 k)
\end{aligned}
$$




$$
\begin{gathered}
\left.+\left(\sum_{j=0}^{2 n} w_{j}{\overline{y_{j}}}^{k-2}\right)\right] z^{k-2 n-1} \\
+\left[\left(\sum_{j=0}^{2 n} u_{j}{\overline{y_{j}}}^{k}\right)\left((2 n+1)^{2}-k^{2}\right)\right. \\
+\left(\sum_{j=0}^{2 n} v_{j}{\overline{y_{j}}}^{k-1}\right)(2 k-1) \\
\left.-\left(\sum_{j=0}^{2 n} w_{j}{\overline{y_{j}}}^{k-2}\right)\right] z^{k} \\
+\frac{-1}{2}\left[\left(\sum_{j=0}^{2 n} u_{j}{\overline{y_{j}}}^{k}\right) k(k-2 n-1)\right. \\
+\left(\sum_{j=0}^{2 n} v_{j}{\overline{y_{j}}}^{k-1}\right)(2 n-2 k+2) \\
\left.\left.+\left(\sum_{j=0}^{2 n} w_{j} \bar{y}_{j}^{k-2}\right)\right] z^{2 n+1+k}\right\} .
\end{gathered}
$$

Corollary 5. (i) If $\left\{z_{j}\right\}_{j=0}^{2 n-1}$ are the $2 n$ roots of 1 , then the Hermite interpolation polynomial satisfying (1) is

$$
\begin{aligned}
\mathscr{H}_{-3 n, 3 n-1}(z) & \\
=\frac{1}{8 n^{3}} & \times \sum_{k=-n}^{n-1}\left\{\frac { 1 } { 2 } \left[\left(\sum_{j=0}^{2 n-1} u_{j} \bar{z}_{j}^{k}\right) k(k+2 n)\right.\right. \\
& -\left(\sum_{j=0}^{2 n-1} v_{j} \bar{z}_{j}^{k-1}\right)(2 n+2 k-1) \\
& \left.+\left(\sum_{j=0}^{2 n-1} w_{j} \bar{z}_{j}^{k-2}\right)\right] z^{k-2 n} \\
+ & {\left[\left(\sum_{j=0}^{2 n-1} u_{j} \bar{z}_{j}^{k}\right)\left(4 n^{2}-k^{2}\right)\right.} \\
& +\left(\sum_{j=0}^{2 n-1} v_{j} \bar{z}_{j}^{k-1}\right)(2 k-1) \\
& \left.-\left(\sum_{j=0}^{2 n-1} w_{j} \bar{z}_{j}^{k-2}\right)\right] z^{k}
\end{aligned}
$$

$$
\begin{aligned}
& +\frac{1}{2}\left[\left(\sum_{j=0}^{2 n-1} u_{j} \bar{z}_{j}^{k}\right) k(k-2 n)\right. \\
& +\left(\sum_{j=0}^{2 n-1} v_{j} \bar{z}_{j}^{k-1}\right)(2 n-2 k+1) \\
& \left.\left.+\left(\sum_{j=0}^{2 n-1} w_{j} \bar{z}_{j}^{k-2}\right)\right] z^{2 n+k}\right\} .
\end{aligned}
$$

(ii) If $\left\{z_{j}\right\}_{j=0}^{2 n}$ are the $2 n+1$ roots of 1 , then the Hermite interpolation polynomial satisfying (1) is

$$
\begin{aligned}
& \mathscr{H}_{-3 n-1,3 n+1}(z) \\
& =\frac{1}{(2 n+1)^{3}} \\
& \times \sum_{k=-n}^{n}\left\{\frac { 1 } { 2 } \left[\left(\sum_{j=0}^{2 n} u_{j} \bar{z}_{j}^{k}\right) k(k+2 n+1)\right.\right. \\
& -\left(\sum_{j=0}^{2 n} v_{j} \bar{z}_{j}^{k-1}\right)(2 n+2 k) \\
& \left.+\left(\sum_{j=0}^{2 n} w_{j} \bar{z}_{j}^{k-2}\right)\right] z^{k-2 n-1} \\
& +\left[\left(\sum_{j=0}^{2 n} u_{j} \bar{z}_{j}^{k}\right)\left((2 n+1)^{2}-k^{2}\right)\right. \\
& +\left(\sum_{j=0}^{2 n} v_{j} \bar{z}_{j}^{k-1}\right)(2 k-1) \\
& \left.-\left(\sum_{j=0}^{2 n} w_{j} \bar{z}_{j}^{k-2}\right)\right] z^{k} \\
& +\frac{1}{2}\left[\left(\sum_{j=0}^{2 n} u_{j} \bar{z}_{j}^{k}\right) k(k-2 n-1)\right. \\
& +\left(\sum_{j=0}^{2 n} v_{j} \bar{z}_{j}^{k-1}\right)(2 n-2 k+2) \\
& \left.\left.+\left(\sum_{j=0}^{2 n} w_{j} \bar{z}_{j}^{k-2}\right)\right] z^{2 n+1+k}\right\} .
\end{aligned}
$$

Remark 6. (a) From (i), (ii), and (iii) in Proposition 3 it is immediate to obtain an expression for the fundamental polynomials of Hermite interpolation. 
(b) Notice that the coefficients of the expressions given before can be computed in an easy and efficient way by using the FFT.

\section{Barycentric Expression}

In this section, our aim is to obtain a barycentric expression for the interpolation polynomial $\mathscr{H}_{-n-E[n / 2], n+E[(n-1) / 2]}(z)$. We distinguish two cases according to the nodal system having an even or odd number of points.

3.1. Nodal System with an Even Number of Points. First we assume that the nodal system has an even number of points that we denote by $2 n$ and we try to obtain the expression of $\mathscr{H}_{-3 n, 3 n-1}(z)$ that we denote for simplicity, $\mathscr{H}(z)$. Since $\mathscr{H}(z)$ can be written in terms of the fundamental polynomials of Hermite interpolation first we obtain suitable expressions for these polynomials.

Lemma 7. The polynomials $\mathscr{E}_{j}(z)=\left(z^{2 n}-\lambda\right)^{3} / z^{3 n}\left(z-\alpha_{j}\right)$, $\mathscr{F}_{j}(z)=\left(z^{2 n}-\lambda\right)^{3} / z^{3 n}\left(z-\alpha_{j}\right)^{2}$, and $\mathscr{G}_{j}(z)=\left(z^{2 n}-\lambda\right)^{3} / z^{3 n}(z-$ $\left.\alpha_{j}\right)^{3}$ for $j=0, \ldots, 2 n-1$ satisfy

(i) $\mathscr{E}_{j}\left(\alpha_{i}\right)=\mathscr{E}_{j}^{\prime}\left(\alpha_{i}\right)=0$, for all $i=0, \ldots, 2 n-1$, $\mathscr{E}_{j}^{\prime \prime}\left(\alpha_{i}\right)=0$, for all $i \neq j$, and $\mathscr{E}_{j}^{\prime \prime}\left(\alpha_{j}\right)=16 \lambda \alpha_{j}^{n} n^{3} / \alpha_{j}^{3}$.

(ii) $\mathscr{F}_{j}\left(\alpha_{i}\right)=0$, for all $i=0, \ldots, 2 n-1, \mathscr{F}_{j}^{\prime}\left(\alpha_{i}\right)=$ 0 , for all $i \neq j, \mathscr{F}_{j}^{\prime}\left(\alpha_{j}\right)=8 \lambda \alpha_{j}^{n} n^{3} / \alpha_{j}^{3}, \mathscr{F}_{j}^{\prime \prime}\left(\alpha_{i}\right)=$ 0 , for all $i \neq j$, and $\mathscr{F}_{j}^{\prime \prime}\left(\alpha_{j}\right)=-\left(24 \lambda \alpha_{j}^{n} n^{3} / \alpha_{j}^{4}\right)$,

(iii) $\mathscr{G}_{j}\left(\alpha_{i}\right)=0$, for all $i \neq j, \mathscr{G}_{j}\left(\alpha_{j}\right)=8 \lambda \alpha_{j}^{n} n^{3} / \alpha_{j}^{3}$, $\mathscr{G}_{j}^{\prime}\left(\alpha_{i}\right)=0$, for all $i \neq j, \quad \mathscr{G}_{j}^{\prime}\left(\alpha_{j}\right)=-\left(12 \lambda \alpha_{j}^{n} n^{3} / \alpha_{j}^{4}\right)$, $\mathscr{G}^{\prime \prime}\left(\alpha_{i}\right)=0$, for all $i \neq j$, and $\mathscr{G}_{j}^{\prime \prime}\left(\alpha_{j}\right)=4 \lambda \alpha_{j}^{n} n^{3}\left(2 n^{2}+\right.$ $7) / \alpha_{j}^{5}$.

Proof. Taking into account that $\left(z^{2 n}-\lambda\right)^{3}=\Pi_{j=0}^{2 n-1}\left(z-\alpha_{j}\right)^{3}$, then it is clear that $\mathscr{E}_{j}\left(\alpha_{j}\right)=\mathscr{E}_{j}^{\prime}\left(\alpha_{j}\right)=0$, for all $i=$ $0, \ldots, 2 n-1, \mathscr{E}_{j}^{\prime \prime}\left(\alpha_{i}\right)=0$, for all $i \neq j$, and $\mathscr{E}_{j}^{\prime \prime}\left(\alpha_{j}\right) \neq 0$. In the same way, it is immediate to see that $\mathscr{F}_{j}\left(\alpha_{i}\right)=0$, for all $i=$ $0, \ldots, 2 n-1, \mathscr{F}_{j}^{\prime}\left(\alpha_{i}\right)=0$ for $i \neq j, \mathscr{F}_{j}^{\prime}\left(\alpha_{j}\right) \neq 0, \mathscr{F}_{j}^{\prime \prime}\left(\alpha_{i}\right)=0$ for $i \neq j$, and $\mathscr{F}_{j}^{\prime \prime}\left(\alpha_{j}\right) \neq 0$. Furthermore, $\mathscr{G}_{j}\left(\alpha_{i}\right)=0$ for $i \neq j$, $\mathscr{G}_{j}\left(\alpha_{j}\right) \neq 0$ and $\mathscr{G}_{j}^{\prime}\left(\alpha_{i}\right)=0$ for $i \neq j, \mathscr{G}_{j}^{\prime}\left(\alpha_{j}\right) \neq 0$ and $\mathscr{G}_{j}^{\prime \prime}\left(\alpha_{i}\right)=$ 0 for $i \neq j$ and $\mathscr{G}_{j}^{\prime \prime}\left(\alpha_{j}\right) \neq 0$.

For obtaining the exact nonvanishing values we proceed as follows.

(i) If we define $e_{j}(y)=\mathscr{E}_{j}\left(\alpha_{j} y\right)$, we obtain $e_{j}(y)=$ $\lambda \alpha_{j}^{n}\left(y^{2 n}-1\right)^{3} / \alpha_{j} y^{3 n}(y-1)$. By taking derivatives and evaluating at $y=1$ we get $e_{j}^{\prime \prime}(1)=16 \lambda \alpha_{j}^{n} n^{3} / \alpha_{j}$, from which we deduce $\mathscr{E}_{j}^{\prime \prime}\left(\alpha_{j}\right)=16 \lambda \alpha_{j}^{n} n^{3} / \alpha_{j}^{3}$.

(ii) In the same way, if we define $f_{j}(y)=\mathscr{F}_{j}\left(\alpha_{j} y\right)$ we get that $f_{j}(y)=\lambda \alpha_{j}^{n}\left(y^{2 n}-1\right)^{3} / \alpha_{j}^{2} y^{3 n}(y-1)^{2}$. By taking derivatives and evaluating at $y=1$ we obtain $f_{j}^{\prime}(1)=8 n^{3} \lambda \alpha_{j}^{n} / \alpha_{j}^{2}$ and $f_{j}^{\prime \prime}(1)=-\left(24 \lambda \alpha_{j}^{n} n^{3} / \alpha_{j}^{2}\right)$, from which we deduce the values of $\mathscr{F}_{j}^{\prime}\left(\alpha_{j}\right)$ and $\mathscr{F}_{j}^{\prime \prime}\left(\alpha_{j}\right)$

(iii) Finally, if we define $g_{j}(y)=\mathscr{G}_{j}\left(\alpha_{j} y\right)$ we get $g_{j}(y)=\lambda \alpha_{j}^{n}\left(y^{2 n}-1\right)^{3} / \alpha_{j}^{3} y^{3 n}(y-1)^{3}$. By evaluating $g_{j}$ and its derivatives at $y=1$ we have that $g_{j}(1)=8 n^{3} \lambda \alpha_{j}^{n} / \alpha_{j}^{3}, g_{j}^{\prime}(1)=-\left(12 n^{3} \lambda \alpha_{j}^{n} / \alpha_{j}^{3}\right)$, and $g_{j}^{\prime \prime}(1)=4 n^{3}\left(2 n^{2}+7\right) \lambda \alpha_{j}^{n} / \alpha_{j}^{3}$, from which we obtain the values of $\mathscr{G}_{j}\left(\alpha_{j}\right), \mathscr{G}_{j}^{\prime}\left(\alpha_{j}\right)$, and $\mathscr{G}_{j}^{\prime \prime}\left(\alpha_{j}\right)$.

Proposition 8. The fundamental polynomials of the Hermite interpolation in the Laurent space $\Lambda_{-3 n, 3 n-1}[z], \mathscr{A}_{j}(z), \mathscr{B}_{j}(z)$, and $\mathscr{C}_{j}(z)$, for $j=0, \ldots, 2 n-1$, characterized by

$$
\begin{array}{r}
\mathscr{A}_{j}\left(\alpha_{i}\right)=\delta_{i, j}, \quad \mathscr{A}_{j}^{\prime}\left(\alpha_{i}\right)=0, \quad \mathscr{A}_{j}^{\prime \prime}\left(\alpha_{i}\right)=0, \\
\forall i=0, \ldots, 2 n-1, \\
\mathscr{B}_{j}\left(\alpha_{i}\right)=0, \quad \mathscr{B}_{j}^{\prime}\left(\alpha_{i}\right)=\delta_{i, j}, \quad \mathscr{B}_{j}^{\prime \prime}\left(\alpha_{i}\right)=0, \\
\forall i=0, \ldots, 2 n-1, \\
\mathscr{C}_{j}\left(\alpha_{i}\right)=0, \quad \mathscr{C}_{j}^{\prime}\left(\alpha_{i}\right)=0, \quad \mathscr{C}_{j}^{\prime \prime}\left(\alpha_{i}\right)=\delta_{i, j}, \\
\forall i=0, \ldots, 2 n-1,
\end{array}
$$

have the following expressions

$$
\begin{aligned}
\mathscr{A}_{j}(z)= & \frac{\alpha_{j}\left(1-n^{2}\right)}{16 \lambda \alpha_{j}^{n} n^{3}} \frac{\left(z^{2 n}-\lambda\right)^{3}}{z^{3 n}\left(z-\alpha_{j}\right)} \\
& +\frac{3 \alpha_{j}^{2}}{16 \lambda \alpha_{j}^{n} n^{3}} \frac{\left(z^{2 n}-\lambda\right)^{3}}{z^{3 n}\left(z-\alpha_{j}\right)^{2}} \\
& +\frac{\alpha_{j}^{3}}{8 \lambda \alpha_{j}^{n} n^{3}} \frac{\left(z^{2 n}-\lambda\right)^{3}}{z^{3 n}\left(z-\alpha_{j}\right)^{3}} \\
\mathscr{B}_{j}(z)= & \frac{3 \alpha_{j}^{2}}{16 \lambda \alpha_{j}^{n} n^{3}} \frac{\left(z^{2 n}-\lambda a\right)^{3}}{z^{3 n}\left(z-\alpha_{j}\right)} \\
& +\frac{\alpha_{j}^{3}}{8 \lambda \alpha_{j}^{n} n^{3}} \frac{\left(z^{2 n}-\lambda\right)^{3}}{z^{3 n}\left(z-\alpha_{j}\right)^{2}}, \\
\mathscr{C}_{j}(z)= & \frac{\alpha_{j}^{3}}{16 \lambda \alpha_{j}^{n} n^{3}} \frac{\left(z^{2 n}-\lambda\right)^{3}}{z^{3 n}\left(z-\alpha_{j}\right)} .
\end{aligned}
$$

Proof. It is clear that $\mathscr{A}_{j}(z), \mathscr{B}_{j}(z)$, and $\mathscr{C}_{j}(z)$ can be written in the following form:

$$
\begin{gathered}
\mathscr{A}_{j}(z)=a_{j, 0} \mathscr{E}_{j}(z)+b_{j, 0} \mathscr{F}_{j}(z)+c_{j, 0} \mathscr{G}_{j}(z), \\
\mathscr{B}_{j}(z)=a_{j, 1} \mathscr{E}_{j}(z)+b_{j, 1} \mathscr{F}_{j}(z), \\
\mathscr{C}_{j}(z)=a_{j, 2} \mathscr{E}_{j}(z),
\end{gathered}
$$

with $\mathscr{E}_{j}(z), \mathscr{F}_{j}(z)$, and $\mathscr{G}_{j}(z)$ given in Lemma 7 . 
To compute $a_{j, 2}$ take into account that it must be $\mathscr{C}_{j}^{\prime \prime}\left(\alpha_{j}\right)=1$. Then applying the preceding lemma we get that $1=a_{j, 2}\left(16 \lambda \alpha_{j}^{n} n^{3} / \alpha_{j}^{3}\right)$, from which it follows that $a_{j, 2}=$ $\alpha_{j}^{3} / 16 \lambda \alpha_{j}^{n} n^{3}$.

For computing $a_{j, 1}$ and $b_{j, 1}$ we use that $\mathscr{B}_{j}^{\prime}\left(\alpha_{j}\right)=1$ and $\mathscr{B}_{j}^{\prime \prime}\left(\alpha_{j}\right)=0$ and we obtain the following system:

$$
\begin{aligned}
& 1=a_{j, 1} \mathscr{E}_{j}^{\prime}\left(\alpha_{j}\right)+b_{j, 1} \mathscr{F}_{j}^{\prime}\left(\alpha_{j}\right), \\
& 0=a_{j, 1} \mathscr{E}_{j}^{\prime \prime}\left(\alpha_{j}\right)+b_{j, 1} \mathscr{F}_{j}^{\prime \prime}\left(\alpha_{j}\right) .
\end{aligned}
$$

By applying Lemma 7 and solving the system we get the result.

Finally, to obtain the coefficients $a_{j, 0}, b_{j, 0}$, and $c_{j, 0}$ in the expression of $\mathscr{A}_{j}(z)$, we proceed in the same way. By applying the interpolation conditions we have the system

$$
\begin{gathered}
0=a_{j, 0} \mathscr{E}_{j}^{\prime \prime}\left(\alpha_{j}\right)+b_{j, 0} \mathscr{F}_{j}^{\prime \prime}\left(\alpha_{j}\right)+c_{j, 0} \mathscr{G}_{j}^{\prime \prime}\left(\alpha_{j}\right), \\
0=b_{j, 0} \mathscr{F}_{j}^{\prime}\left(\alpha_{j}\right)+c_{j, 0} \mathscr{G}_{j}^{\prime}\left(\alpha_{j}\right), \\
1=c_{j, 0} \mathscr{G}_{j}\left(\alpha_{j}\right) .
\end{gathered}
$$

Then, by using Lemma 7 and solving the system we conclude our result.

It is straightforward to deduce, from the preceding Proposition 8, the so-called barycentric expression for $\mathscr{H}(z)$.

Proposition 9. (i) The polynomial $\mathscr{H}_{0}(z) \in \Lambda_{-3 n, 3 n-1}[z]$ satisfying the conditions $\mathscr{H}_{0}\left(\alpha_{j}\right)=u_{j}, \mathscr{H}_{0}^{\prime}\left(\alpha_{j}\right)=0$, and $\mathscr{H}_{0}^{\prime \prime}\left(\alpha_{j}\right)=0$, for all $j=0, \ldots, 2 n-1$, has the following barycentric expression:

$\mathscr{H}_{0}(z)$

$$
\begin{aligned}
= & \sum_{j=0}^{2 n-1} \frac{u_{j}}{\alpha_{j}^{n}}\left[\frac{\alpha_{j}^{3}}{\left(z-\alpha_{j}\right)^{3}}+\frac{3 \alpha_{j}^{2}}{2\left(z-\alpha_{j}\right)^{2}}+\frac{\alpha_{j}\left(1-n^{2}\right)}{2\left(z-\alpha_{j}\right)}\right] \\
& \times\left(\sum_{j=0}^{2 n-1} \frac{1}{\alpha_{j}^{n}}\left[\frac{\alpha_{j}^{3}}{\left(z-\alpha_{j}\right)^{3}}+\frac{3 \alpha_{j}^{2}}{2\left(z-\alpha_{j}\right)^{2}}+\frac{\alpha_{j}\left(1-n^{2}\right)}{2\left(z-\alpha_{j}\right)}\right]\right)^{-1} .
\end{aligned}
$$

(ii) The polynomial $\mathscr{H}_{1}(z) \in \Lambda_{-3 n, 3 n-1}[z]$ satisfying the conditions $\mathscr{H}_{1}\left(\alpha_{j}\right)=0, \mathscr{H}_{1}^{\prime}\left(\alpha_{j}\right)=v_{j}$, and $\mathscr{H}_{1}^{\prime \prime}\left(\alpha_{j}\right)=$ 0 , for all $j=0, \ldots, 2 n-1$, has the following barycentric expression:

$$
\begin{aligned}
\mathscr{H}_{1}(z) & =\sum_{j=0}^{2 n-1} \frac{v_{j}}{\alpha_{j}^{n}}\left[\frac{\alpha_{j}^{3}}{\left(z-\alpha_{j}\right)^{2}}+\frac{3 \alpha_{j}^{2}}{2\left(z-\alpha_{j}\right)}\right] \\
& \times\left(\sum_{j=0}^{2 n-1} \frac{1}{\alpha_{j}^{n}}\left[\frac{\alpha_{j}^{3}}{\left(z-\alpha_{j}\right)^{3}}+\frac{3 \alpha_{j}^{2}}{2\left(z-\alpha_{j}\right)^{2}}+\frac{\alpha_{j}\left(1-n^{2}\right)}{2\left(z-\alpha_{j}\right)}\right]\right)^{-1} .
\end{aligned}
$$

(iii) The polynomial $\mathscr{H}_{2}(z) \in \Lambda_{-3 n, 3 n-1}[z]$ satisfying the conditions $\mathscr{H}_{2}\left(\alpha_{j}\right)=0, \mathscr{H}_{2}^{\prime}\left(\alpha_{j}\right)=0$, and $\mathscr{H}_{2}^{\prime \prime}\left(\alpha_{j}\right)=$ $w_{j}$, for all $j=0, \ldots, 2 n-1$, has the following barycentric expression:

$$
\begin{aligned}
\mathscr{H}_{2} & (z) \\
= & \sum_{j=0}^{2 n-1} \frac{w_{j}}{\alpha_{j}^{n}}\left[\frac{\alpha_{j}^{3}}{2\left(z-\alpha_{j}\right)}\right] \\
& \times\left(\sum_{j=0}^{2 n-1} \frac{1}{\alpha_{j}^{n}}\left[\frac{\alpha_{j}^{3}}{\left(z-\alpha_{j}\right)^{3}}+\frac{3 \alpha_{j}^{2}}{2\left(z-\alpha_{j}\right)^{2}}+\frac{\alpha_{j}\left(1-n^{2}\right)}{2\left(z-\alpha_{j}\right)}\right]\right)^{-1} .
\end{aligned}
$$

(iv) The polynomial $\mathscr{H}(z) \in \Lambda_{-3 n, 3 n-1}[z]$ satisfying (1) has the following barycentric expression:

$$
\begin{aligned}
& \qquad \sum_{j=0}^{\mathscr{H}(z)} \frac{1}{\alpha_{j}^{n}}\left[u_{j}\left(\frac{\alpha_{j}^{3}}{\left(z-\alpha_{j}\right)^{3}}+\frac{3 \alpha_{j}^{2}}{2\left(z-\alpha_{j}\right)^{2}}+\frac{\alpha_{j}\left(1-n^{2}\right)}{2\left(z-\alpha_{j}\right)}\right)\right. \\
& +v_{j}\left(\frac{\alpha_{j}^{3}}{\left(z-\alpha_{j}\right)^{2}}+\frac{3 \alpha_{j}^{2}}{2\left(z-\alpha_{j}\right)}\right) \\
& \left.\quad+w_{j}\left(\frac{\alpha_{j}^{3}}{2\left(z-\alpha_{j}\right)}\right)\right] \\
& \quad \times\left(\sum_{j=0}^{2 n-1} \frac{1}{\alpha_{j}^{n}}\left[\frac{\alpha_{j}^{3}}{\left(z-\alpha_{j}\right)^{3}}+\frac{3 \alpha_{j}^{2}}{2\left(z-\alpha_{j}\right)^{2}}+\frac{\alpha_{j}\left(1-n^{2}\right)}{2\left(z-\alpha_{j}\right)}\right]\right)^{-1} .
\end{aligned}
$$

Proof. (i) It is immediate if we take into account that $\mathscr{H}_{0}(z)=$ $\sum_{j=0}^{2 n-1} u_{j} \mathscr{A}_{j}(z)$, with $\mathscr{A}_{j}(z)$ given in Proposition 8. Thus, if we divide the polynomial by $1=\sum_{j=0}^{2 n-1} \mathscr{A}_{j}(z)$, that is,

$$
\mathscr{H}_{0}(z)=\frac{\sum_{j=0}^{2 n-1} u_{j} \mathscr{A}_{j}(z)}{\sum_{j=0}^{2 n-1} \mathscr{A}_{j}(z)},
$$

and we use the expressions of $\mathscr{A}_{j}(z)$, after doing some simplifications, we obtain the result.

(ii) Take into account that $\mathscr{H}_{1}(z)=\sum_{j=0}^{2 n-1} v_{j} \mathscr{B}_{j}(z)$, with $\mathscr{B}_{j}(z)$ given in Proposition 8 and proceed in the same way as in (i).

(iii) Take into account that $\mathscr{H}_{2}(z)=\sum_{j=0}^{2 n-1} w_{j} \mathscr{C}_{j}(z)$, with $\mathscr{C}_{j}(z)$ given in Proposition 8 and proceed in the same way as in (i).

(iv) Take into account that

$$
\mathscr{H}(z)=\sum_{j=0}^{2 n-1}\left(u_{j} \mathscr{A}_{j}(z)+v_{j} \mathscr{B}_{j}(z)+w_{j} \mathscr{C}_{j}(z)\right),
$$


with $\mathscr{A}_{j}(z), \mathscr{B}_{j}(z)$, and $\mathscr{C}_{j}(z)$ given in Proposition 8 and proceed in the same way as in (i).

3.2. Nodal System with an Odd Number of Points. Now we assume that the nodal system $\left\{\alpha_{j}\right\}_{j=0}^{2 n}$ is constituted by the $2 n+1$ roots of $\lambda$, with $|\lambda|=1$. In this case we obtain the barycentric expression for the Laurent polynomial of Hermite interpolation $\mathscr{H}_{-3 n, 3 n+1}(z)$ that we denote by $\mathscr{H}(z)$, characterized by

$$
\mathscr{H}\left(\alpha_{j}\right)=u_{j}, \quad \mathscr{H}^{\prime}\left(\alpha_{j}\right)=v_{j}, \quad \mathscr{H}^{\prime \prime}\left(\alpha_{j}\right)=w_{j},
$$

for $j=0, \ldots, 2 n$,

where $\left\{u_{j}\right\}_{j=0}^{2 n},\left\{v_{j}\right\}_{j=0}^{2 n}$ and $\left\{w_{j}\right\}_{j=0}^{2 n}$ are fixed complex numbers.

Proceeding like in the previous case, first we obtain the following auxiliary results.

Lemma 10. The Laurent polynomials, $\mathscr{E}_{j}(z)=\left(z^{2 n+1}-\lambda\right)^{3} /$ $z^{3 n+1}\left(z-\alpha_{j}\right), \mathscr{F}_{j}(z)=\left(z^{2 n+1}-\lambda\right)^{3} / z^{3 n+1}\left(z-\alpha_{j}\right)^{2}$, and $\mathscr{G}_{j}$ $(z)=\left(z^{2 n+1}-\lambda\right)^{3} / z^{3 n+1}\left(z-\alpha_{j}\right)^{3}$, for $j=0, \cdots, 2 n$, satisfy

(i) $\mathscr{E}_{j}\left(\alpha_{i}\right)=0, \mathscr{E}_{j}^{\prime}\left(\alpha_{i}\right)=0$, for all $i=0, \ldots$, $2 n, \quad \mathscr{E}_{j}^{\prime \prime}\left(\alpha_{i}\right)=0$, for all $i \neq j$, and $\mathscr{E}_{j}^{\prime \prime}\left(\alpha_{j}\right)=$ $2 \lambda \alpha_{j}^{n}(2 n+1)^{3} / \alpha_{j}^{2}$,

(ii) $\mathscr{F}_{j}\left(\alpha_{i}\right)=0$, for all $i=0, \ldots, 2 n, \mathscr{F}_{j}^{\prime}\left(\alpha_{i}\right)=$ 0 , for all $i \neq j, \mathscr{F}_{j}^{\prime}\left(\alpha_{j}\right)=\lambda \alpha_{j}^{n}(2 n+1)^{3} / \alpha_{j}^{2}, \mathscr{F}_{j}^{\prime \prime}\left(\alpha_{i}\right)=$ 0 , for all $i \neq j$, and $\mathscr{F}_{j}^{\prime \prime}\left(\alpha_{j}\right)=-\left(2 \lambda \alpha_{j}^{n}(2 n+1)^{3} / \alpha_{j}^{3}\right)$,

(iii) $\mathscr{G}_{j}\left(\alpha_{i}\right)=0$, for all $i \neq j, \mathscr{G}_{j}\left(\alpha_{j}\right)=\lambda \alpha_{j}^{n}(2 n+1)^{3} /$ $\alpha_{j}^{2}, \mathscr{G}_{j}^{\prime}\left(\alpha_{i}\right)=0$, for all $i \neq j, \mathscr{G}_{j}^{\prime}\left(\alpha_{j}\right)=-\left(\lambda \alpha_{j}^{n}\right.$ $\left.(2 n+1)^{3} / \alpha_{j}^{3}\right), \mathscr{G}_{j}^{\prime \prime}\left(\alpha_{i}\right)=0$, for all $i \neq j$, and $\mathscr{G}_{j}^{\prime \prime}$ $\left(\alpha_{j}\right)=\left(\lambda \alpha_{j}^{n}(2 n+1)^{3}\left(n^{2}+n+2\right)\right) / \alpha_{j}^{4}$.

Proof. It is easy to prove the result following the same steps of the proof of the preceding Lemma 7.

Proposition 11. The fundamental polynomials of Hermite interpolation in the Laurent space $\Lambda_{-3 n-1,3 n+1}[z], \mathscr{A}_{j}(z) \mathscr{B}_{j}(z)$ $y \mathscr{C}_{j}(z)$, for $j=0, \ldots, 2 n$, characterized by

$$
\begin{array}{lrr}
\mathscr{A}_{j}\left(\alpha_{i}\right)=\delta_{i, j}, & \mathscr{A}_{j}^{\prime}\left(\alpha_{i}\right)=0, & \mathscr{A}_{j}^{\prime \prime}\left(\alpha_{i}\right)=0, \\
& \forall i=0, \ldots, 2 n, \\
\mathscr{B}_{j}\left(\alpha_{i}\right)=0, & \mathscr{B}_{j}^{\prime}\left(\alpha_{i}\right)=\delta_{i, j}, & \mathscr{B}_{j}^{\prime \prime}\left(\alpha_{i}\right)=0, \\
& \forall i=0, \ldots, 2 n, \\
\mathscr{C}_{j}\left(\alpha_{i}\right)=0, & \mathscr{C}_{j}^{\prime}\left(\alpha_{i}\right)=0, \quad \mathscr{C}_{j}^{\prime \prime}\left(\alpha_{i}\right)=\delta_{i, j}, \\
& \forall i=0, \ldots, 2 n,
\end{array}
$$

have the following expressions:

$$
\begin{aligned}
\mathscr{A}_{j}(z)= & \frac{\alpha_{j}^{2}}{\lambda \alpha_{j}^{n}(2 n+1)^{3}} \frac{\left(z^{2 n+1}-\lambda\right)^{3}}{z^{3 n+1}\left(z-\alpha_{j}\right)^{3}} \\
& +\frac{\alpha_{j}}{\lambda \alpha_{j}^{n}(2 n+1)^{3}} \frac{\left(z^{2 n+1}-\lambda\right)^{3}}{z^{3 n+1}\left(z-\alpha_{j}\right)^{2}} \\
& -\frac{\left(n^{2}+n\right)}{2 \lambda \alpha_{j}^{n}(2 n+1)^{3}} \frac{\left(z^{2 n+1}-\lambda\right)^{3}}{z^{3 n+1}\left(z-\alpha_{j}\right)}, \\
\mathscr{B}_{j}(z)= & \frac{\alpha_{j}^{2}}{\lambda \alpha_{j}^{n}(2 n+1)^{3}} \frac{\left(z^{2 n+1}-\lambda\right)^{3}}{z^{3 n+1}\left(z-\alpha_{j}\right)^{2}} \\
& +\frac{\alpha_{j}}{\lambda \alpha_{j}^{n}(2 n+1)^{3}} \frac{\left(z^{2 n+1}-\lambda\right)^{3}}{z^{3 n+1}\left(z-\alpha_{j}\right)}, \\
\mathscr{C}_{j}(z)= & \frac{\alpha_{j}^{2}}{2 \lambda \alpha_{j}^{n}(2 n+1)^{3}} \frac{\left(z^{2 n+1}-\lambda\right)^{3}}{z^{3 n+1}\left(z-\alpha_{j}\right)} .
\end{aligned}
$$

Proof. It is similar to the proof of Proposition 8.

It is straightforward to deduce, from the preceding Proposition, the so-called barycentric expression for $\mathscr{H}(z)$.

Proposition 12. (i) The polynomial $\mathscr{H}_{0}(z) \in \Lambda_{-3 n-1,3 n+1}[z]$ satisfying the conditions $\mathscr{H}_{0}\left(\alpha_{j}\right)=u_{j}, \mathscr{H}_{0}^{\prime}\left(\alpha_{j}\right)=0$, and $\mathscr{H}_{0}^{\prime \prime}\left(\alpha_{j}\right)=0$, for all $j=0, \ldots, 2 n$, has the barycentric expression

$$
\begin{aligned}
\mathscr{H}_{0}(z) & \\
= & \sum_{j=0}^{2 n} \frac{u_{j}}{\alpha_{j}^{n}}\left[\frac{\alpha_{j}^{2}}{\left(z-\alpha_{j}\right)^{3}}+\frac{\alpha_{j}}{\left(z-\alpha_{j}\right)^{2}}-\frac{\left(n^{2}+n\right)}{2\left(z-\alpha_{j}\right)}\right] \\
& \times\left(\sum_{j=0}^{2 n} \frac{1}{\alpha_{j}^{n}}\left[\frac{\alpha_{j}^{2}}{\left(z-\alpha_{j}\right)^{3}}+\frac{\alpha_{j}}{\left(z-\alpha_{j}\right)^{2}}-\frac{\left(n^{2}+n\right)}{2\left(z-\alpha_{j}\right)}\right]\right)^{-1} .
\end{aligned}
$$

(ii) The polynomial $\mathscr{H}_{1}(z) \in \Lambda_{-3 n-1,3 n+1}[z]$ satisfying the conditions $\mathscr{H}_{1}\left(\alpha_{j}\right)=0, \mathscr{H}_{1}^{\prime}\left(\alpha_{j}\right)=v_{j}$, and $\mathscr{H}_{1}^{\prime \prime}\left(\alpha_{j}\right)=0$, for all $j=0, \ldots, 2 n$, has the barycentric expression

$$
\begin{aligned}
\mathscr{H}_{1}(z) & \\
= & \sum_{j=0}^{2 n} \frac{v_{j}}{\alpha_{j}^{n}}\left[\frac{\alpha_{j}^{2}}{\left(z-\alpha_{j}\right)^{2}}+\frac{\alpha_{j}}{\left(z-\alpha_{j}\right)}\right] \\
& \times\left(\sum_{j=0}^{2 n} \frac{1}{\alpha_{j}^{n}}\left[\frac{\alpha_{j}^{2}}{\left(z-\alpha_{j}\right)^{3}}+\frac{\alpha_{j}}{\left(z-\alpha_{j}\right)^{2}}-\frac{\left(n^{2}+n\right)}{2\left(z-\alpha_{j}\right)}\right]\right)^{-1} .
\end{aligned}
$$


(iii) The polynomial $\mathscr{H}_{2}(z) \in \Lambda_{-3 n-1,3 n+1}[z]$ satisfying the conditions $\mathscr{H}_{2}\left(\alpha_{j}\right)=0, \mathscr{H}_{2}^{\prime}\left(\alpha_{j}\right)=0$, and $\mathscr{H}_{2}^{\prime \prime}\left(\alpha_{j}\right)=w_{j}$, for all $j=0, \ldots, 2 n$, has the barycentric expression

$$
\begin{aligned}
\mathscr{H}_{2}(z) & \\
= & \sum_{j=0}^{2 n} \frac{w_{j}}{\alpha_{j}^{n}}\left[\frac{\alpha_{j}^{2}}{2\left(z-\alpha_{j}\right)}\right] \\
& \times\left(\sum_{j=0}^{2 n} \frac{1}{\alpha_{j}^{n}}\left[\frac{\alpha_{j}^{2}}{\left(z-\alpha_{j}\right)^{3}}+\frac{\alpha_{j}}{\left(z-\alpha_{j}\right)^{2}}-\frac{\left(n^{2}+n\right)}{2\left(z-\alpha_{j}\right)}\right]\right)^{-1} .
\end{aligned}
$$

(iv) The polynomial $\mathscr{H}(z) \in \Lambda_{-3 n-1,3 n+1}[z]$ satisfying the conditions (42) has the barycentric expression

$\mathscr{H}(z)$

$$
\begin{aligned}
& =\sum_{j=0}^{2 n} \frac{1}{\alpha_{j}^{n}}\left[u_{j}\left(\frac{\alpha_{j}^{2}}{\left(z-\alpha_{j}\right)^{3}}+\frac{\alpha_{j}}{\left(z-\alpha_{j}\right)^{2}}-\frac{\left(n^{2}+n\right)}{2\left(z-\alpha_{j}\right)}\right)\right. \\
& +v_{j}\left(\frac{\alpha_{j}^{2}}{\left(z-\alpha_{j}\right)^{2}}+\frac{\alpha_{j}}{\left(z-\alpha_{j}\right)}\right) \\
& \left.+w_{j}\left(\frac{\alpha_{j}^{2}}{2\left(z-\alpha_{j}\right)}\right) \frac{1}{\alpha_{j}^{n}}\right] \\
& \quad \times\left(\sum_{j=0}^{2 n} \frac{1}{\alpha_{j}^{n}}\left[\frac{\alpha_{j}^{2}}{\left(z-\alpha_{j}\right)^{3}}+\frac{\alpha_{j}}{\left(z-\alpha_{j}\right)^{2}}-\frac{\left(n^{2}+n\right)}{2\left(z-\alpha_{j}\right)}\right]\right)^{-1} .
\end{aligned}
$$

Proof. It is similar to the proof of Proposition 9.

Remark 13. Notice the novelty of the barycentric expressions that we have obtained for Hermite interpolation on the unit circle using up to the second derivative. These formulas can be implemented for use in a simple way and they are very useful for computations due to their stability.

\section{Conflict of Interests}

The authors declare that there is no conflict of interests regarding the publication of this paper.

\section{Acknowledgment}

The research was supported by Ministerio de Educación y Ciencia of Spain under Grant no. MTM2011-22713.

\section{References}

[1] L. Daruis and P. González-Vera, "A note on Hermite-Fejér interpolation for the unit circle," Applied Mathematics Letters, vol. 14, no. 8, pp. 997-1003, 2001.
[2] P. J. Davis, Interpolation and Approximation, Dover, New York, NY, USA, 1975.

[3] J. Szabados and P. Vértesi, Interpolation of Functions, World Scientific, Singapore, 1990.

[4] E. Berriochoa, A. Cachafeiro, and E. M. Martínez Brey, "Some improvements to the Hermite-Fejér interpolation on the circle and bounded interval," Computers and Mathematics with Applications, vol. 61, no. 4, pp. 1228-1240, 2011.

[5] E. Berriochoa, A. Cachafeiro, J. Díaz, and E. Martínez Brey, "Rate of convergence of Hermite-Fejér interpolation on the unit circle," Journal of Applied Mathematics, vol. 2013, Article ID 407128, 8 pages, 2013.

[6] E. Berriochoa and A. Cachafeiro, "Algorithms for solving Hermite interpolation problems using the Fast Fourier Transform," Journal of Computational and Applied Mathematics, vol. 235, no. 4, pp. 882-894, 2010.

[7] E. Berriochoa, A. Cachafeiro, J. Díaz, and J. Illán, "Two algorithms for Hermite interpolation on the circle and applications," submitted.

[8] P. Henrici, Essentials of Numerical Analysis with Pocket Calculator Demonstrations, John Wiley \& Sons, New York, NY, USA, 1982.

[9] S. Bahadur, "(0,0,1) interpolation on the unit circle," International Journal of Mathematical Analysis, vol. 5, no. 29, pp. 14291434, 2011.

[10] W. Chen and A. Sharma, "Lacunary interpolation on some non-uniformly distributed nodes on the unit circle," Annales Universitatis Scientiarum Budapestinensis, Sectio Mathematica, vol. 16, pp. 69-82, 2004.

[11] J. L. Walsh, Interpolation and Approximation by Rational Functions in the Complex Plane, vol. 20 of American Mathematical Society: Colloquium Publications, American Mathematical Society, Providence, RI, USA, 5th edition, 1969.

[12] N. J. Higham, "The numerical stability of barycentric Lagrange interpolation," IMA Journal of Numerical Analysis, vol. 24, no. 4, pp. 547-556, 2004. 


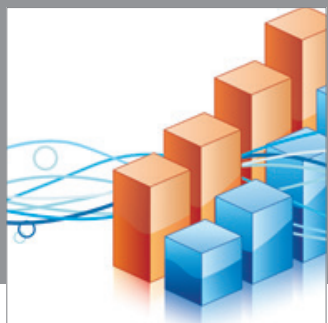

Advances in

Operations Research

mansans

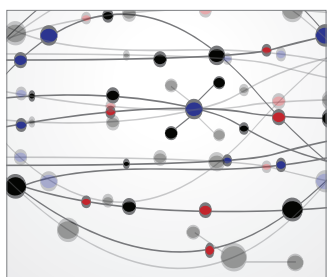

The Scientific World Journal
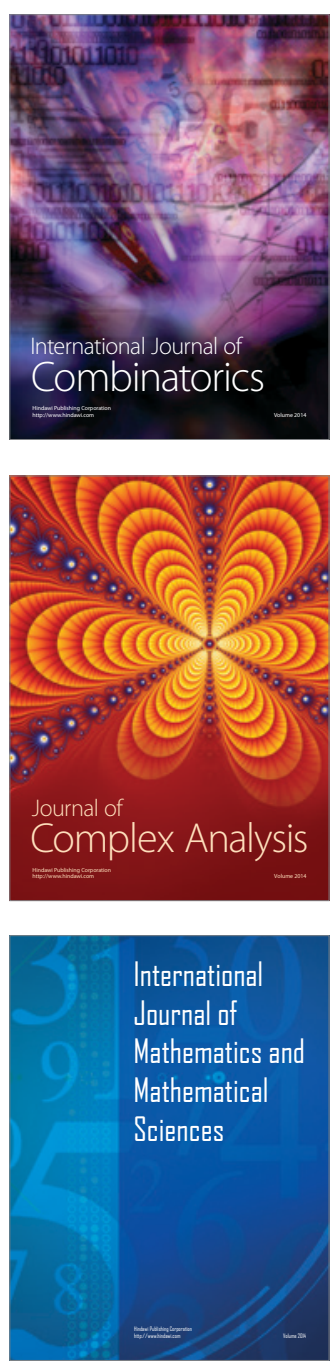
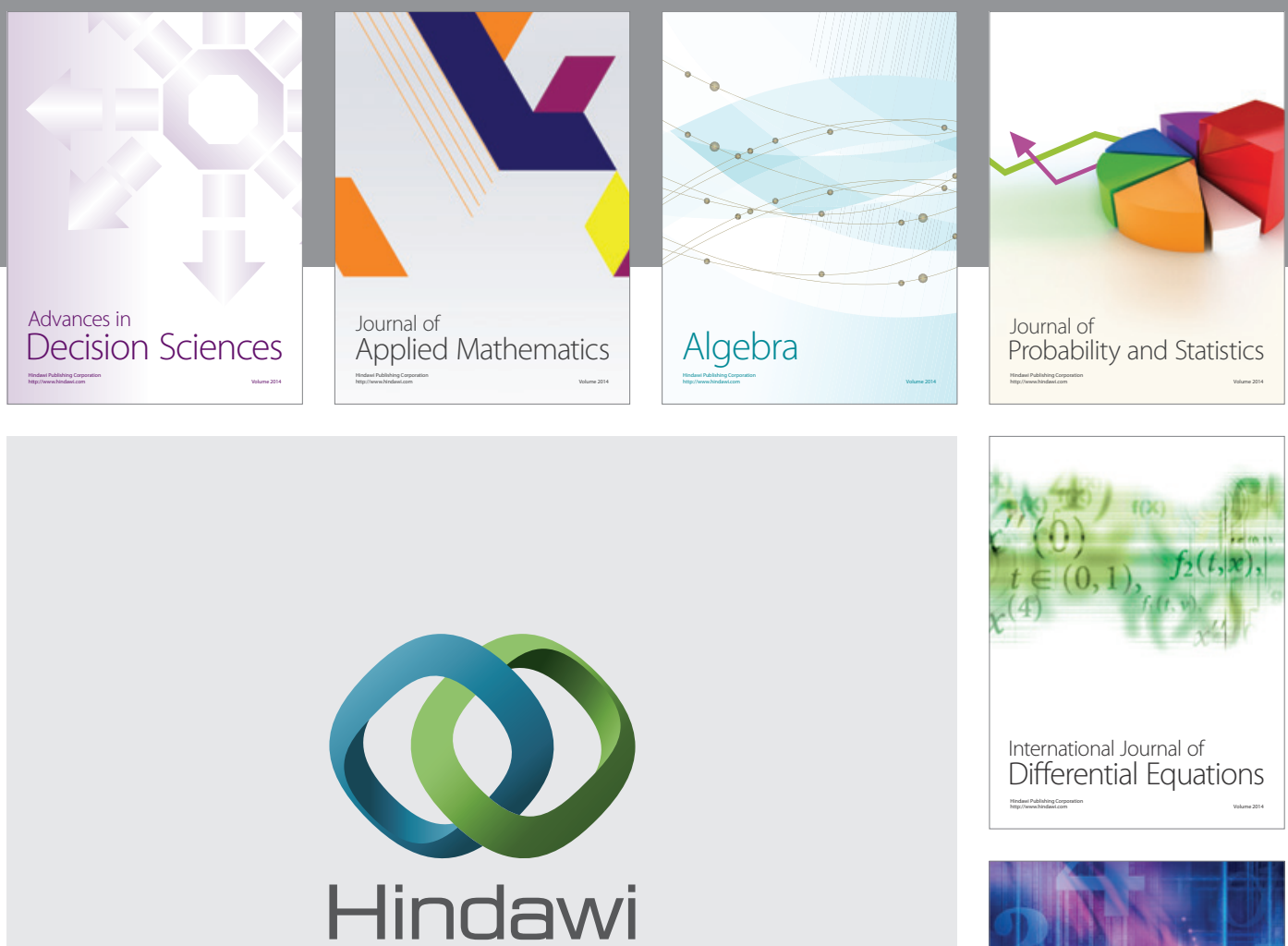

Submit your manuscripts at http://www.hindawi.com
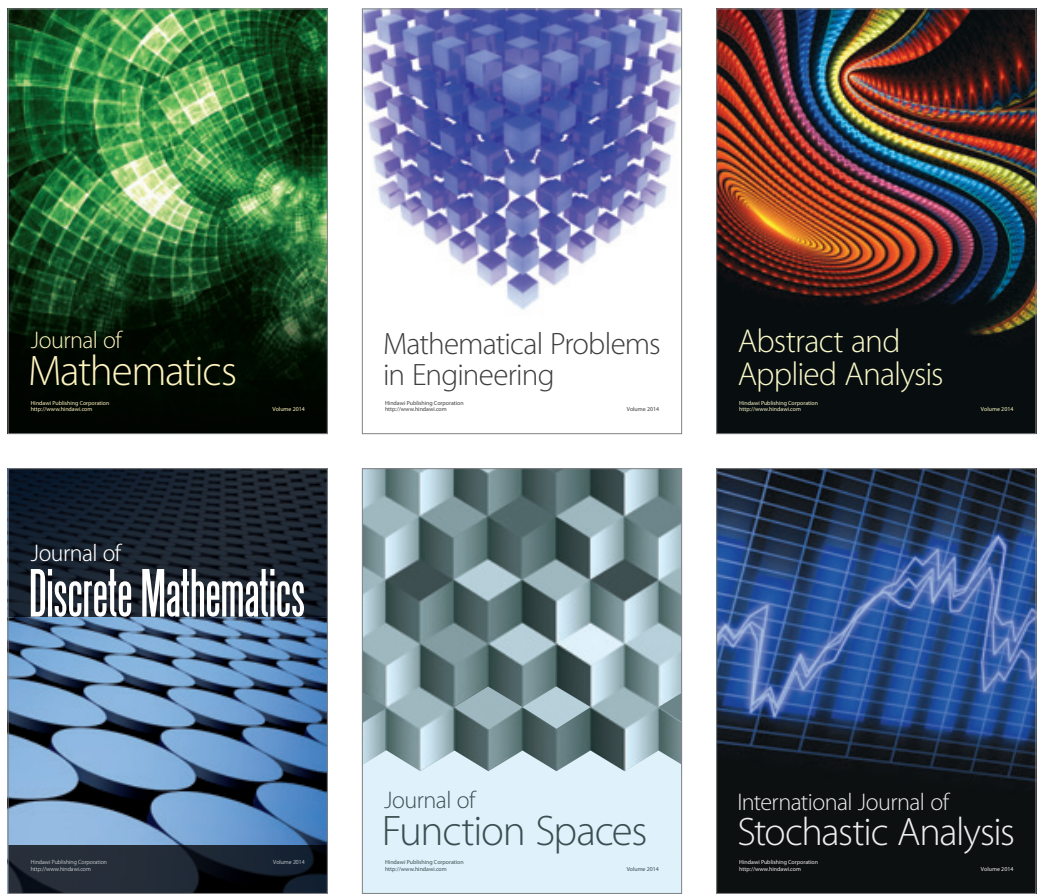

Journal of

Function Spaces

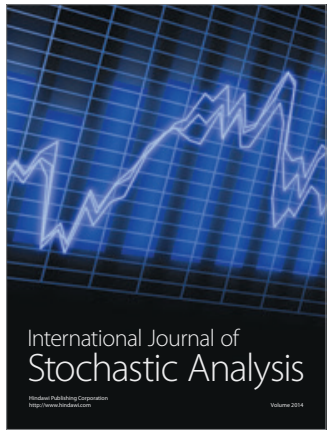

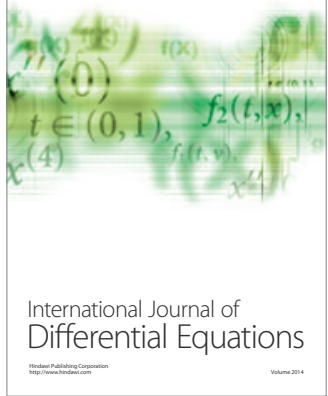
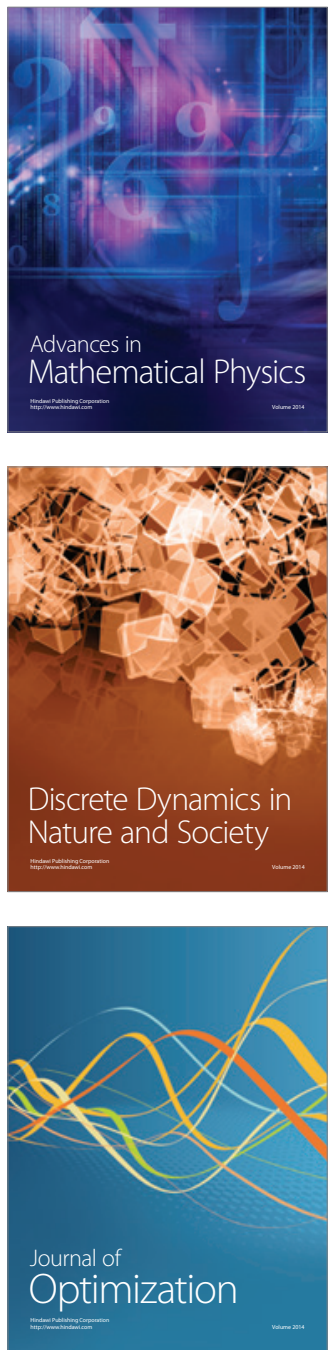